\title{
Robust Observer Design for an Isolated Power System with Model Uncertainty using $H_{\infty}$-Norm
}

\author{
Tomonori Goya*, Tomonobu Senjyu ${ }^{\dagger}$, Eitaro Omine*, \\ Atsushi Yona*, Naomitsu Urasaki*, and Toshihisa Funabashi** \\ ${ }^{\dagger *}$ Dept. of Electrical and Electronics Eng., Faculty of Eng., University of the Ryukyus, Okinawa, Japan \\ ** Meidensha Corporation, Tokyo, Japan
}

\begin{abstract}
The output power fluctuations of renewable energy power plants such as wind turbine generators and photovoltaic systems result in frequency deviations and terminal voltage fluctuations. Furthermore, these power fluctuations also affect the turbine shaftings of diesel generators and gas-turbine generators which are the main power generation systems on isolated islands. Therefore, it is important to achieve torsional torque suppression. Since the measurement of torsional torque is technically difficult, and there is an uncertainty in the mechanical constants of the shaft torsional system. This paper presents an estimation system that estimates torsional torque by using a developed $H_{\infty}$ observer. In addition to the above functions, the proposed shaft torque observer incorporates a parameter identification system that aims to improve the estimation accuracy. The simulation results validate the effectiveness of the proposed $H_{\infty}$ observer and the parameter identification.
\end{abstract}

Key Words: Decentralized generators, $H_{\infty}$ observer, Microgrid, Parameter identification, Torsional torque suppression, Wind turbine

\section{INTRODUCTION}

The demand for energy on isolated islands is steadily increasing. At present, power generation on remote islands mostly depends on diesel generators and gas-turbine generators. Gas-turbine generators have been introduced on isolated islands because of their superiority to diesel generators in reducing emissions of $\mathrm{NOx}$ and $\mathrm{CO}_{2}$ and also because of their compact size. Due to the fossil fuel combustion in gas-turbine generators, they suffer from many associated problems such as the scarcity of fossil fuels, environmental pollution, the cost of fuel transportation, and so on.

One of the solutions for these issues is to introduce renewable energy power plants such as photovoltaic systems and wind turbine generators, which use clean energy that is abundantly available in nature. However, the output power of wind turbines is not constant but varies proportionately with the cube of the wind speed. This significantly affects the stability of isolated power systems resulting in frequency deviations and voltage fluctuations. Since isolated island power systems are weak, these disturbances become serious problems. Therefore, it is necessary to compensate the load variations and output power fluctuations of wind turbine generators by regulating the output power from gas turbine generators. However, the excessive power deviations of loads and wind

\footnotetext{
Manuscript received Jan. 22, 2010; revised Jun. 27, 2010

$\dagger$ Corresponding Author: b985542@ tec.u-ryukyu.ac.jp

Tel:+81-98-895-8686, Fax:+81-98-895-8686, Ryukyus Univ.

* Dept. of Electrical and Electronics Eng., Ryukyus Univ., Japan

** Meidensha Corporation, Japan
}

turbine generators affect the turbine shafting, that is, the torsional torque oscillations [1], [2]. Although gas-turbine generators have a shear-pin that breaks when excessive torque is applied to the turbine shafts, once the shear-pin breaks to protect the turbine shaft, it requires a lot of time to repair and restart the generators. Therefore, there has been great demand for control schemes to suppress torsional torque oscillations to achieve stable generator operation. In [3], an excitation control system for achieving torsional torque suppression is proposed. However, since the control performance of the excitation controller is greatly dependent on the observation accuracy of the torsional torque, a shaft torque observer that achieves accurate estimation is necessary.

Some researchers have applied linear quadratic regulator (LQR) theory to the observer design for torsional torque estimation [3]. In the case of a linear system without parameter errors or measurement noise, this observer system can achieve good estimation accuracy of the torsional torque. Nevertheless, in the cases of parameter uncertainty in the system and the existence of noise in the measured values, the estimation accuracy of the torsional torque is not satisfactory. Given this factor, we apply a $H_{\infty}$ observer for torsional torque estimation, which achieves robust estimation against system uncertainties like parameter variations and modeling errors. Also, the proposed $H_{\infty}$ observation system can be designed in the frequency domain, which gives the desired frequency characteristics to reduce the gain against the measurement noise. In addition to the above functions, the proposed shaft torque observer incorporates a parameter identification system that 


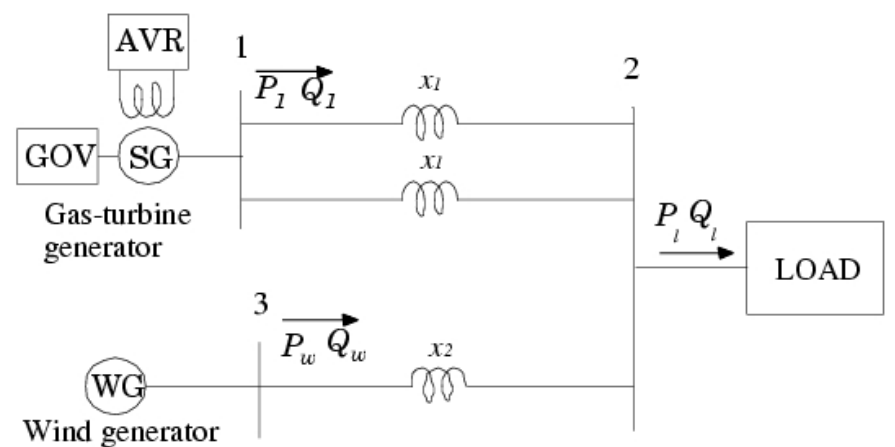

Fig. 1. Isolated power system model.

TABLE I

SYSTEM CONSTANTS

$$
\begin{gathered}
\text { Gas-turbine generator } \\
H=1.07 \mathrm{~s}, x_{d}=1.5 \mathrm{pu}, x_{d}^{\prime}=0.30 \mathrm{pu}, x_{q}=1.06 \mathrm{pu} \\
x_{q}^{\prime}=0.3 \mathrm{pu}, T_{d 0}^{\prime}=3.7 \mathrm{~s}, T_{q 0}^{\prime}=0.21 \mathrm{~s} \\
K_{A}=50, K_{G}=100, T_{G}=4 \mathrm{~s}, T_{A}=0.06 \mathrm{~s} \\
\text { Transmission lines } \\
x_{1}=0.8 \mathrm{pu}, x_{2}=0.3 \mathrm{pu}
\end{gathered}
$$

aims to improve the estimation accuracy. This paper presents a control strategy for achieving both stabilization control of a remote power system and a torsional torque suppression based on the estimated torsional torque by using a $H_{\infty}$ observer with a parameter identification system. The proposed control system incorporates the $H_{\infty}$ control theory which improves the loadfollowing capability and the robustness of the controller. The effectiveness of the proposed control system is validated by numerical simulation results in MATLAB.

\section{Modeling}

Fig. 1 shows an isolated power system model consisting of a gas-turbine generator, a wind turbine generator, and a load. The reference capacity per unit system is selected as 5MVA and the wind turbine generator is rated at 1.25MVA. The gasturbine generator is modeled as a synchronous generator [12]. The parameter values for each element are shown in Table I. The excitation and the governing system can generally be described as the first-order lag system shown in Figs. 2 and 3 . In this paper, these excitation and governing systems are defined as a conventional control system. The turbine shaft model of a gas-turbine generator can be described as a 7 mass model, as shown in Fig. 4, and is composed of a high-pressure turbine, a generator, an exciter and some joints [5]. For a detailed analysis, this paper adopts a 7 mass torsional system. The mechanical constants of the shaft system are shown in Table 2. The frequency response of the 7 mass torsional system is depicted as a dotted line in Fig. 5. From Fig. 5, it can be seen that there are resonance points in the oscillation mode of the power system (Mode 0) and in the torsional oscillation mode (Mode 1). In designing a controller that achieves a damping of power system oscillations and torsional torque suppression, it is necessary to eliminate these resonance points.

\section{OBSERVER DESIGN}

This section describes the configuration of the generalized plant of the $H_{\infty}$ observer and the selection of the weighting

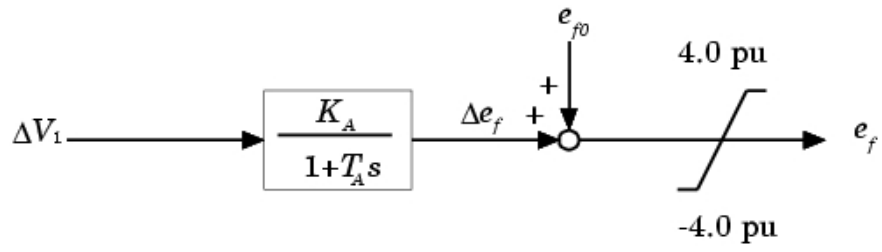

Fig. 2. Automatic voltage regulator.

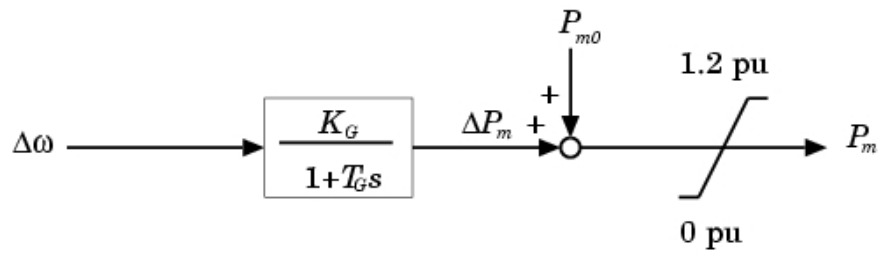

Fig. 3. Governor.

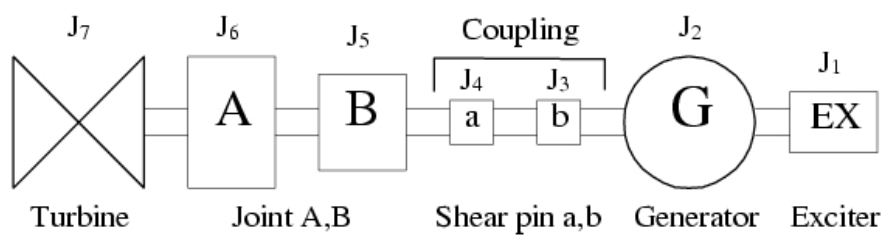

Fig. 4. Turbine shaft model.

function, which determines the performance of the observer system. A typical configuration for a Luenberger observer is shown in Fig. 6. Since it is particularly difficult to get the exact parameters for a real system, there is a parameter error in the state space matrices $\left(A_{o}, B_{o}, C_{o}\right)$ of the model $P_{o b s}(s)$ in the observer system. Furthermore, if there is noise at the measurement point, it will degrade the estimation accuracy. Therefore it is important to include the disturbances due to parameter uncertainty and measurement noise in defining the generalized plant of the $H_{\infty}$ observer. According to [6], the parameter variation can be expressed by the feedback type uncertainty shown in Fig. 7. The measurement noise can be regarded as an inclusion of a high frequency disturbance on the measurement point. The generalized plant of the $H_{\infty}$ observer is defined and shown in Fig. 8. Thus, to minimize the sensitivity to disturbances from parameter uncertainty and measurement noise in the estimation result, the design problem is expressed as the follows:

$$
\min _{\gamma}\left\|\begin{array}{l}
W_{1} T_{w \rightarrow \hat{y}} \\
W_{2} T_{\tilde{n} \rightarrow \hat{y}}
\end{array}\right\|_{\infty} \leq \gamma
$$

where $T_{w \rightarrow \hat{y}}$ and $T_{\tilde{n} \rightarrow \hat{y}}$ are the transfer functions from the disturbance $w$ and the measurement noise $\tilde{n}$ in the estimation result $\hat{y}$ respectively. From the above equation, the design purpose of the $H_{\infty}$ observer is to minimize the $H_{\infty}$ norm of the weighted transfer function from the disturbances caused by parameter uncertainty and measurement noise in the estimation result. In Eq. (1), $W_{1}$ and $W_{2}$ are the weighting functions for the transfer functions $T_{w \rightarrow \hat{y}}$ and $T_{\tilde{n} \rightarrow \hat{y}}$ respectively. The weighting function $W_{1}$ is the weight on the estimated value $\hat{y}$ that decides the robustness of the observer system. Since the disturbances caused by parameter variations will affect 


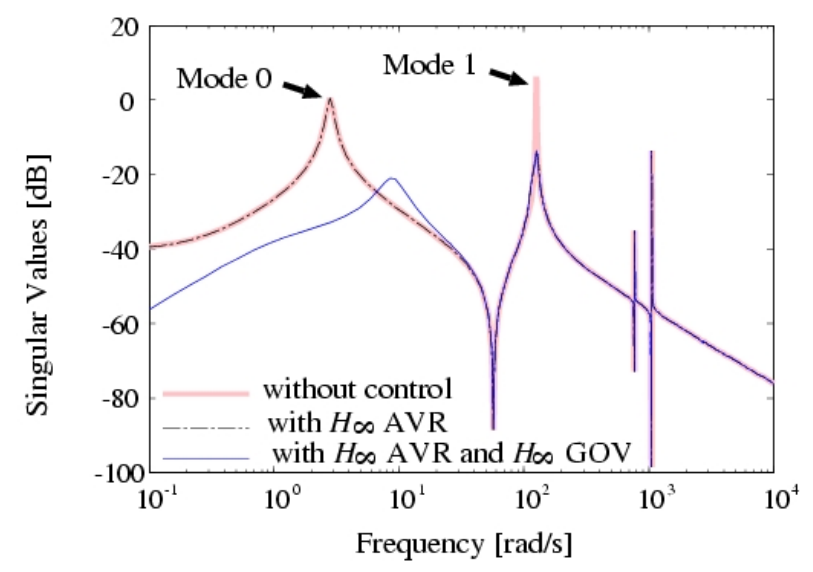

Fig. 5. Frequency response of 7 mass model $\left(T_{e} \rightarrow \omega_{2}\right)$.

TABLE II

MECHANICAL SYSTEM CONSTANTS

\begin{tabular}{|c|c|c|}
\hline Mass & $\begin{array}{c}\text { Inertia constant } \\
H[\mathrm{~s}]\end{array}$ & $\begin{array}{c}\text { Spring constant } \\
K[\mathrm{pu} / \mathrm{rad}]\end{array}$ \\
\hline \hline Turbine & 1.19 & 164.5228 \\
\hline Joint A & 0.0454 & 712.153 \\
\hline Joint B & 0.013 & 300.3748 \\
\hline shearpin a & 0.0025 & 158.5285 \\
\hline shearpin b & 0.0025 & 34.0054 \\
\hline Generator & 0.3185 & 26.0130 \\
\hline Exciter & 0.0044 & \\
\hline
\end{tabular}

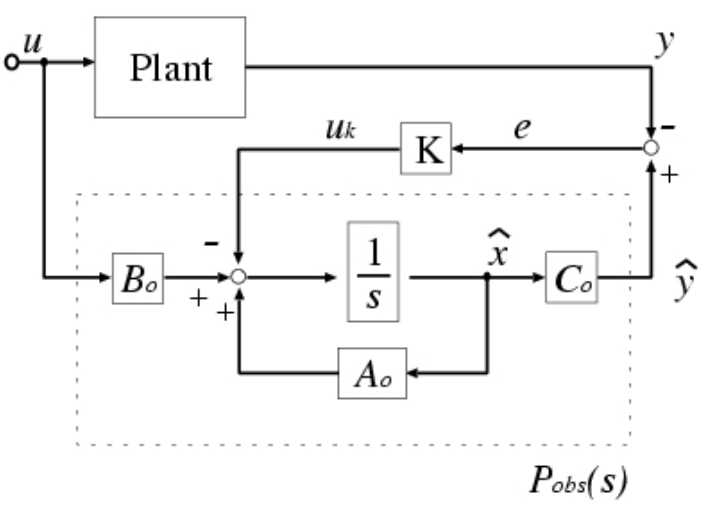

Fig. 6. Plant and observer model.

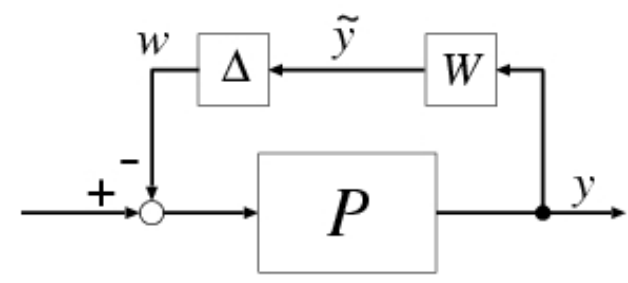

Fig. 7. Multiplicative input uncertainty.

the low frequency component of the frequency characteristic of the system, the weighting function $W_{1}$ is selected to set the high gain in the low frequency domain. The weighting function $W_{2}$ is the weight on the measurement noise. Since the measurement noise can be regarded as a disturbance that mainly has high frequency components, the weighting function $W_{2}$ is selected to have a high gain in the high frequency domain. The singular value plots of the weighting functions

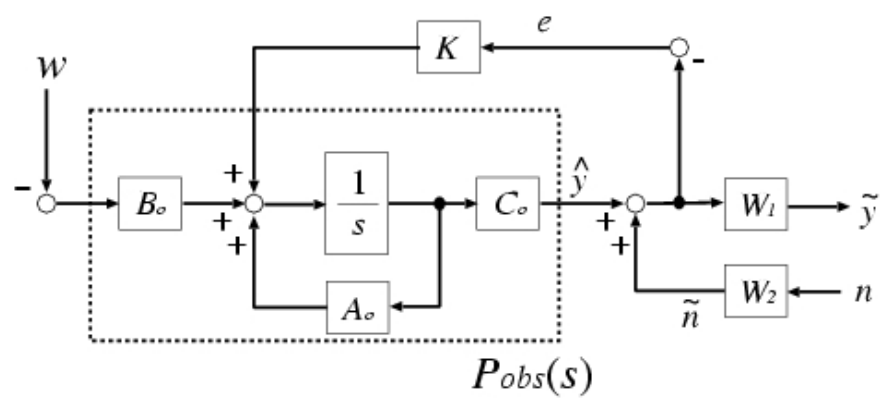

Fig. 8. Generalized plant of $H_{\infty}$ observer.

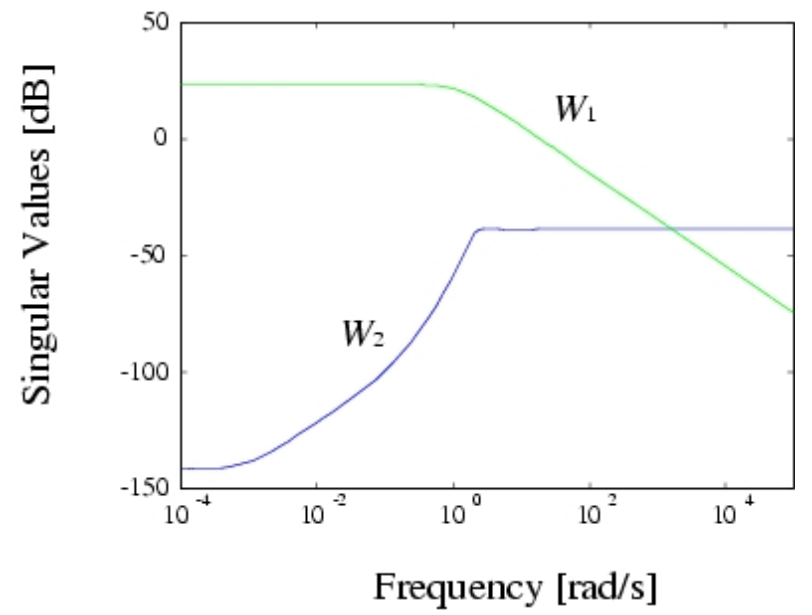

Fig. 9. Singular value plots of weighting functions.

$W_{1}$ and $W_{2}$ are shown in Fig. 9. By using these weighting functions, the $H_{\infty}$ observer is designed based on the LMI (Linear Matrix Inequality) approach [7]-[10].

\section{VERIFICATION OF ESTIMATION ACCURACY}

This section verifies the estimation accuracy of the proposed $H_{\infty}$ observer when compared with a conventional LQR observer.

\section{A. Verification of the Frequency Characteristics}

This subsection compares the frequency characteristics of the $H_{\infty}$ observer and the LQR observer. The LQR observer has two design parameters: the weighting matrices $Q$ and $R$. By increasing the weighting matrix $Q$, the estimation accuracy is improved but the high sensitivity to noise remains. On the other hand, by increasing the weighting matrix $R$, the sensitivity to noise is lowered but the estimation accuracy is degraded. The LQR observer is designed taking into consideration the above trade off. The design procedure of the LQR observer can be found in [6]. The frequency characteristics of the designed LQR observer and the proposed $H_{\infty}$ observer are shown in Fig. 10. Fig. 10(a) shows the singular value plots from the measured value $y$ to the estimated value $\hat{y}$, where the weighting matrices of the LQR observer are set to be $Q=10^{2} I_{q}$ and $R=1$, and $I_{q}$ is an identity matrix of the order $4 \times 4$. From Fig. 10(a), it can be seen that the $H_{\infty}$ observer has a wider bandwidth than the LQR observer, which enables the $H_{\infty}$ observer to achieve accurate estimation. 


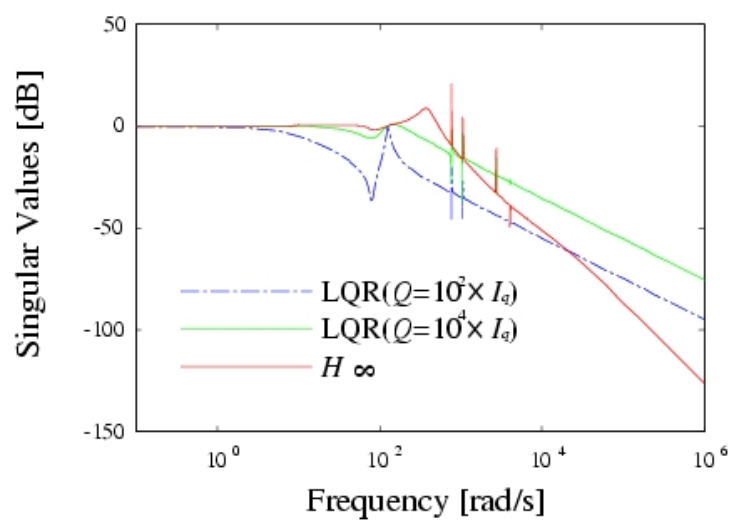

(a) Transfer function $T_{y \rightarrow \tilde{y}}$.

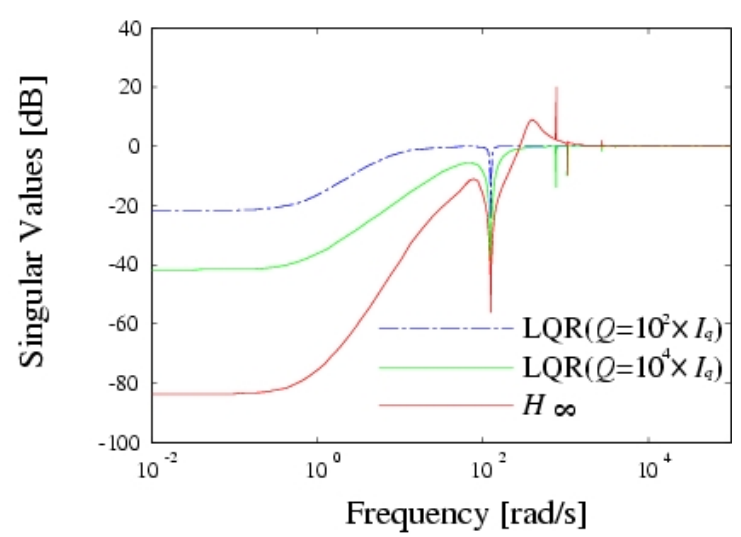

(b) Sensitivity function $T_{y \rightarrow e}$.

Fig. 10. Frequency characteristic of observer system.

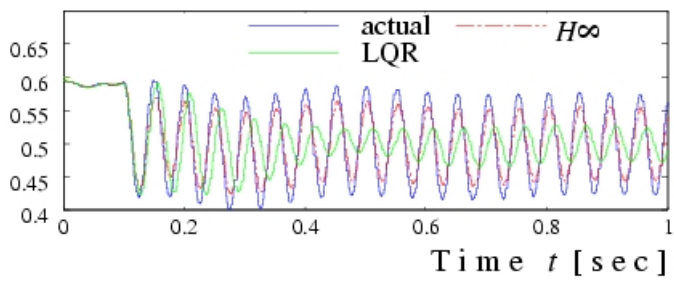

Fig. 11. Estimation results $(\Delta K=-20 \%)$.

The LQR observer can achieve a bandwidth equal to the $H_{\infty}$ observer by increasing the weighting matrix $Q$. However, the observer system also increases the sensitivity to measurement noise by increasing the gain in the high frequency domain. On the other hand, the $H_{\infty}$ observer reduces the gain in the high frequency domain while maintaining a wide bandwidth. Fig. 10(b) shows the singular value plots from the measured value $y$ to the estimation error $e$. Fig. 10(b) implies that the LQR observer is not effective for accurate estimation because the steady state gains of the error dynamics are not sufficiently small resulting in the existence of a steady state error. On the other hand, it is seen that a gain reduction of the error dynamics in the low frequency domain is achieved by using the $H_{\infty}$ observer.

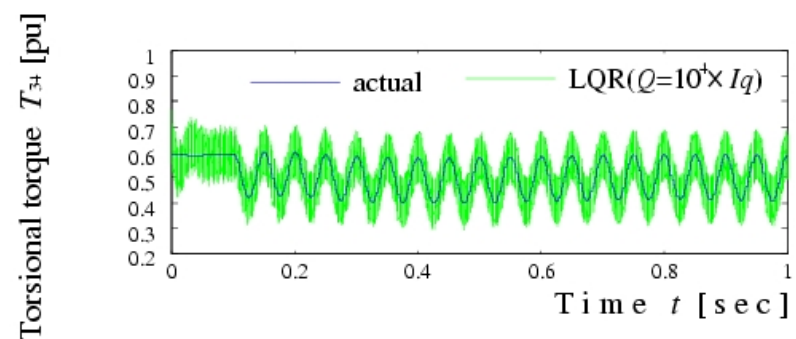

(a) Using LQR observer.

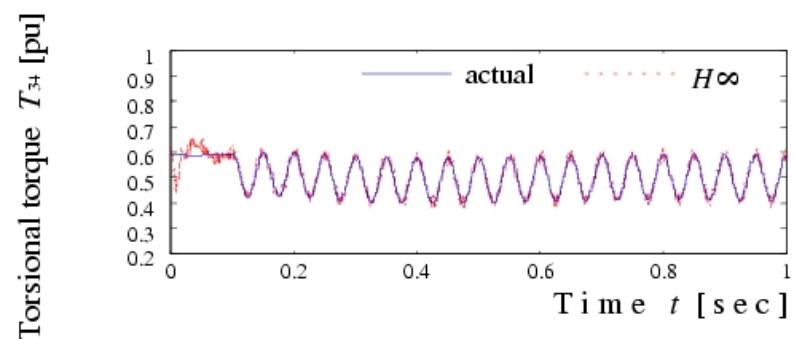

(b) Using $H_{\infty}$ observer.

Fig. 12. Estimation results(in case of noise injection).

\section{B. Robustness Against Parameter Variation and Noise}

This subsection verifies the robustness of the proposed $H_{\infty}$ observer against parameter variation and noise effects. Fig. 11 shows the estimation result by using the LQR observer and the $H_{\infty}$ observer, respectively. In this simulation, we assumed that a parameter error of $-20 \%$ exists in the spring constant $K$ of each section. From the simulation results, the proposed $H_{\infty}$ observer achieves robust estimations against parameter errors while the estimation results using the LQR observer demonstrate insufficient accuracy for use with parameter uncertainty. The LQR observer can achieve robust estimation by increasing the weighting matrix $Q$, but it also has a high sensitivity to measurement noise. Fig. 12 shows the estimation results in the case of a noise injection where the weighting function of the LQR observer is modified to be $Q=10^{4} I_{q}$. From Fig. 12, it is observed that the estimation accuracy of the LQR observer is affected by the measurement noise. Meanwhile, the proposed $H_{\infty}$ observer maintains good estimation accuracy since it is designed to have reduced gain in the high frequency domain. From the above simulation results, it can be seen that the proposed $H_{\infty}$ observer has robustness against both parameter variations and measurement noise.

\section{PARAMETER IDENTIFICATION}

This section presents the parameter identification system for the torsional torque observer that aims for superior estimation accuracy. The typical parameters for a turbine shaft system are the inertia constants of each of the rotating masses, the damping coefficients and the spring constants of the shaft. Though the damping coefficients vary according to the operating conditions, they do not influence the characteristics of the torsional system, the maximum torsional torque or the torsional modal frequency. On the other hand, the characteristics of the torsional system are mostly influenced by the inertia constants and the spring constants of the shaft system. One of the characteristics of the torsional system, that 


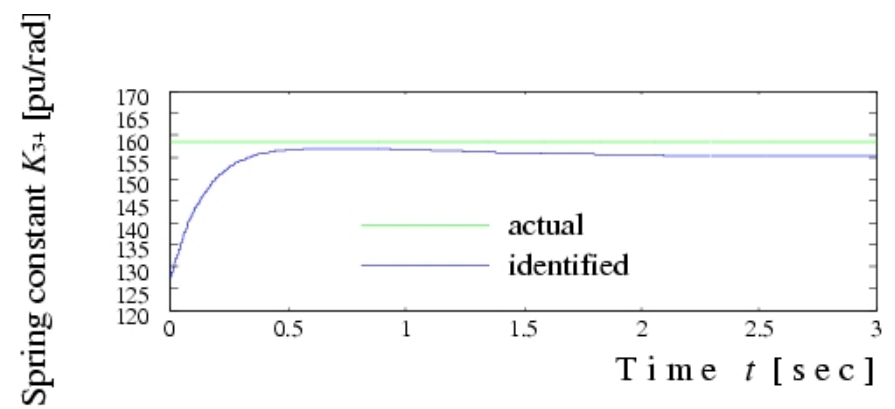

Fig. 13. Parameter identification results.

is the maximum torsional torque, is considerably influenced by the inertia constants. The other characteristic, that is the modal frequency, is mostly influenced by the spring constant of the shaft. For accurate estimation of the torsional torque, the most important thing is to match the phase of the actual and the estimated torsional torques. Thus, the influence of the parameter errors of the spring constants that determine the torsional modal frequency should be considered, hence the need to identify the shaft spring constants. In this paper, the identification system for the shaft parameter, that is the spring constant, is incorporated into the developed $H_{\infty}$ observer for accurate estimation. The fixed gain adaptive identification method is applied to the identification system. From Fig. 4, the torsional torque applied to the shaft between the shear-pin $a \cdot b$ is expressed by the following equation:

$$
\dot{T}_{34}=\omega_{0}\left(\omega_{4}-\omega_{3}\right) \cdot K_{34} .
$$

After multiplying by the state variable filter $\frac{1}{T_{1} s+1}$, the above equation can be summarized as follows:

$$
y=Z^{T} \alpha
$$

where, $y, Z$, and $\alpha$ are defined as follows:

$$
\begin{aligned}
y & =\frac{1}{T_{1} s+1} \dot{T}_{34} \\
Z & =\frac{1}{T_{1} s+1}\left(\omega_{0}\left(\omega_{4}-\omega_{3}\right)\right) \\
\alpha & =K_{34} .
\end{aligned}
$$

The adaptive identification model is defined by the following equation:

$$
\hat{y}=Z^{T} \hat{\alpha}
$$

Thus, the parameter identification system is formulated as below:

$$
\dot{\hat{\alpha}}=-P Z(\hat{y}-y)
$$

where $P$ is the adaptive gain of the identification system. The other spring constants of different sections can be similarly identified with the above described identification procedure. The identification result obtained using the estimated value

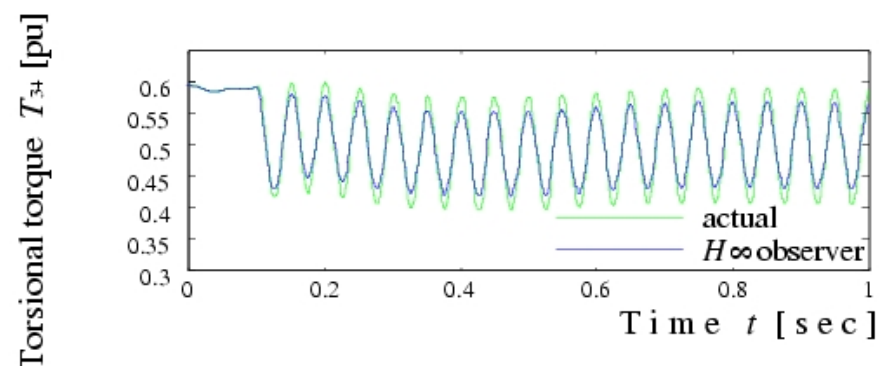

(a) Without parameter identification.

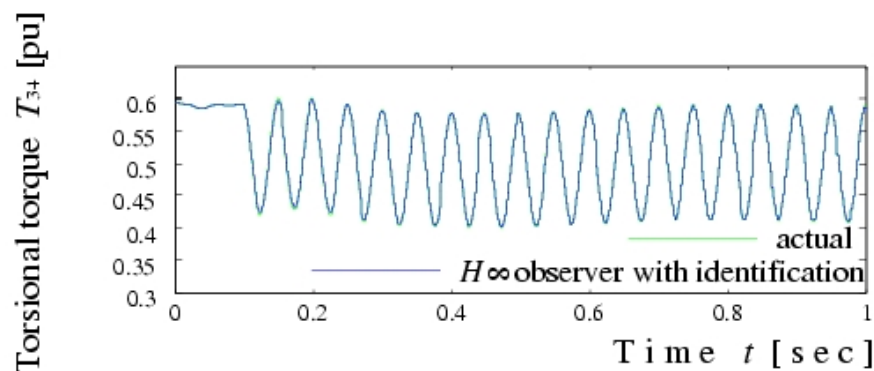

(b) With parameter identification.

Fig. 14. Estimation results with parameter identification.

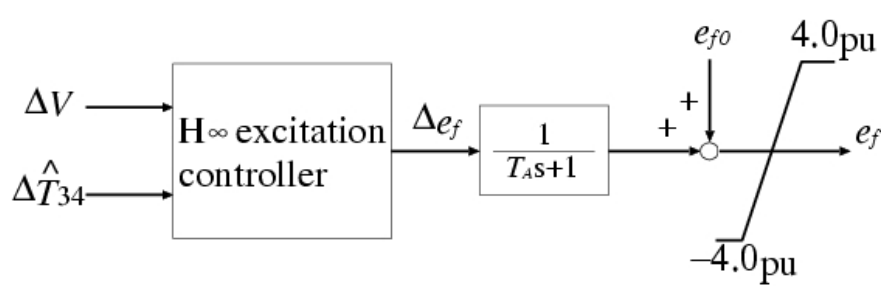

Fig. 15. Configuration of $H_{\infty}$ excitation controller.

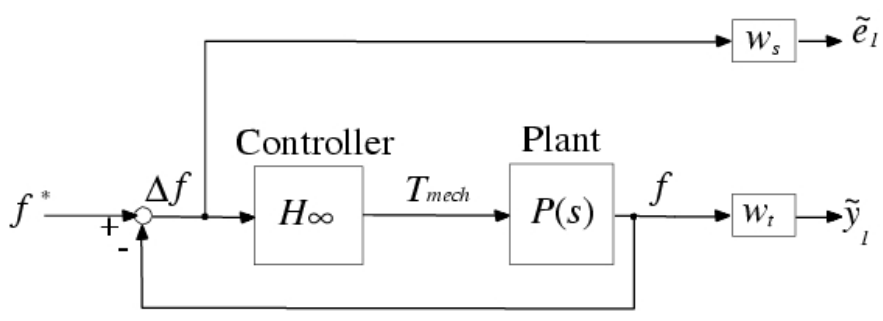

Fig. 16. Configuration of $H_{\infty}$ controller for governor system.

of the $H_{\infty}$ observer is shown in Fig. 13. From Fig. 13, it can be seen that the parameter identification is successfully achieved by using the estimated torsional torque. Fig. 14 shows the estimation results by using the $H_{\infty}$ observer with the identification system. By including the parameter identification system in the $H_{\infty}$ observer, better estimation accuracy can be obtained.

\section{CONTROLLER DESIGN}

This section presents the principles of designing the proposed control system, which achieves torsional torque suppression and power system stabilization. The proposed control system operates as a decentralized control system since the $H_{\infty}$ controllers are designed at the exciter and the governor system individually. Since this paper puts emphasis only on designing the observer system, the design of the $H_{\infty}$ controller 
is briefly explained. The details of designing the $H_{\infty}$ controller can be found in [11]. Fig. 15 shows the configuration of the $H_{\infty}$ excitation controller for achieving terminal voltage control and torsional torque suppression. The controller inputs are the terminal voltage deviation $\Delta V$ and the estimated torsional torque deviation, $\Delta \hat{T}_{34}$, by means of the $H_{\infty}$ observer. The frequency characteristic of the torsional system with the $H_{\infty}$ excitation controller is shown in Fig. 5. From Fig. 5, it can be observed that the elimination of the resonance point of the torsional oscillation mode (Mode 1) is achieved. However, the resonance point representing the power system oscillation mode (Mode 0) still remains. Therefore, the $H_{\infty}$ controller for the governing system should be designed to eliminate the resonance of the power system oscillation in order to achieve stabilization control of the power system. The control system that achieves the damping of the power system oscillation is shown in Fig. 16. The controller adjusts the mechanical input to the gas-turbine generator according to the frequency deviation $\Delta f$ in order to achieve load frequency control and power system stabilization. The frequency response of the torsional system in the case of applying the $H_{\infty}$ governor system is shown as a solid line in Fig. 5. From Fig. 5, it can be seen that the removal of the resonance point of the power system oscillating mode (Mode 0) is successfully achieved.

\section{SIMULATION RESULTS}

In order to verify the effectiveness of the proposed control and observer system, computer simulations are conducted assuming the load deviations and output power fluctuations of wind turbine generator. This simulation assumes parameter errors of $-15 \%$ for each spring constant of the shaft. Simulation results are shown in Fig. 17. From Fig. 17(a), it is observed that load demand is rapidly changed at $t=5 \mathrm{~s}$ and $t=10 \mathrm{~s}$. Fig. 17(b) shows the generating power of the wind turbine generator. These power fluctuations cause frequency deviations and affect the turbine shafting of the gas-turbine generator. Fig. 17(c) shows the torsional torque between the shear-pin $a \cdot b$ experienced by excessive load deviations. From Fig. 17(c), it can be seen that successful torsional torque suppression is achieved by using the proposed $H_{\infty}$ observer system as a result of accurate estimation of torsional torque. Fig. 17(d) shows terminal voltage of the gas-turbine generator. Although the terminal voltage fluctuates to suppress the torsional torque oscillation, the voltage deviation is controlled to be within the acceptable range of $\pm 5 \%$. Fig. 17(e) shows the mechanical input to the gas-turbine generator. From Fig. 17(e), it can be observed that the load-following capability is improved and the torque oscillation is damped by applying the $H_{\infty}$ control to the governor system. From Fig. 17(f), it can be seen that the load frequency control and the damping of the power system oscillations are achieved by applying the proposed control system.

\section{CONCLUSIONS}

This paper proposes an estimation system that estimates the torsional torque by using the developed $H_{\infty}$ observer, which achieves robust estimation of torsional torque against

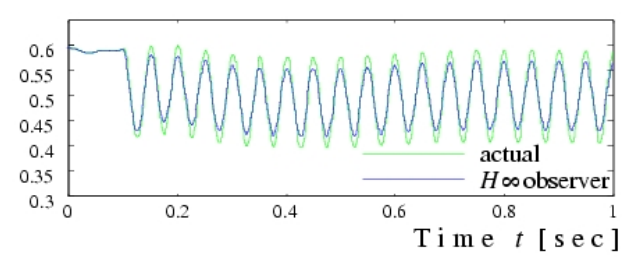

(a) Active power demand, $P_{l}$.

(b) Generating power of wind turbine generator, $P_{w}$.

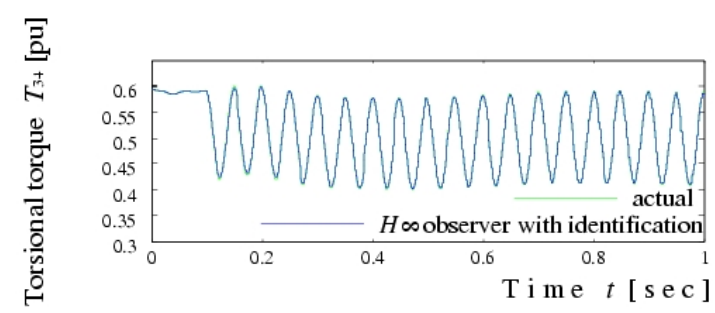

(c) Torsional torque, $T_{34}$.

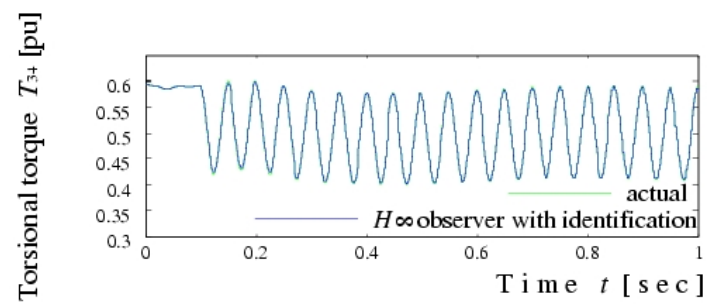

(d) Terminal voltage of bus $1, V_{1}$.

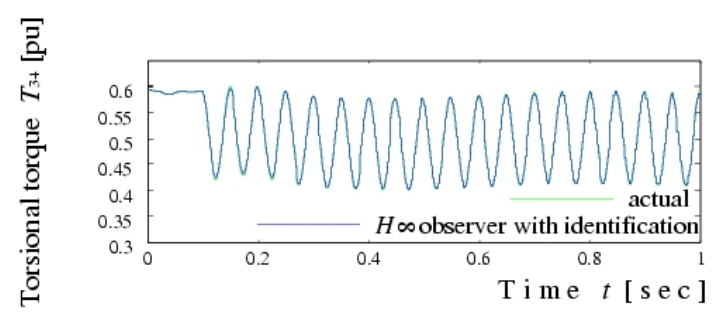

(e) Mechanical input to gas-turbine generator, $T_{m e c h}$.

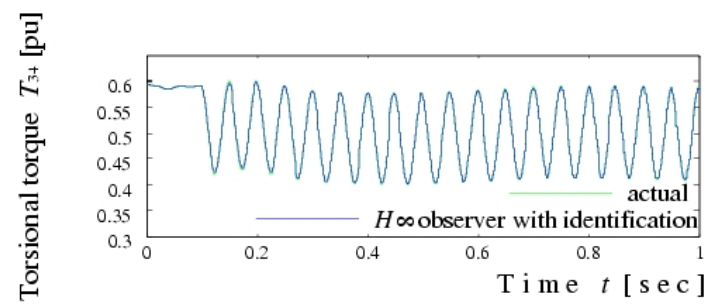

(f) Frequency deviation, $\Delta f$.

Fig. 17. Simulation results $(\Delta K:-15 \%)$.

parameter errors and measurement noise when compared with the LQR observer. By introducing the parameter identification system into the $H_{\infty}$ system, more accurate estimation can 
be achieved. Simulation results validate the effectiveness of the proposed $H_{\infty}$ observer and the parameter identification system.

\section{REFERENCES}

[1] John S. Joyce and Dietrich Lambrecht, "Status of evaluating the fatigue of large stem turbine-generators caused by electrical disturbances," IEEE Trans. Power Apparatus and Systems, vol. PAS-99, No. 1, pp. 111-119, Jan./Feb. 1996.

[2] T. Funabashi, H. Otoguro, G. Fujita, K. Koyanagi, and R. Yokoyama, "An influence of voltage sag duration on non-utility generator's shaft torque," in Proc. IEEE PES'OO Winter Meeting, pp. 153-158, 2000.

[3] T. Kakinoki, R. Yokoyama, G. Fujita, K. Koyanagi, and T Funabashi, "Evaluation technique of turbine shaft fatigue in private-generation systems using consumption rate of shaft life cycle," in Proc. IEEEPowerCon'02 Conf., pp. 779-783, 2002.

[4] Chee-Mun Ong, Dynamic simulation of electric machinery: using Matlab/Simulink, N.J.: Prentice Hall PTR, 1998.

[5] P. Kundur, Power system stability and control, New York: McGrawHill, 1994.

[6] S. Skogestad and I. Postlethwaite, Multivariable feedback control analysis and design, John Wiley and Sons Ltd., 1996.

[7] M. Chilali and P. Gahinet, " $H_{\infty}$ design with pole placement constraints: an LMI approach," IEEE Trans. Automatic Control, Vol. 41, No. 3, pp. 358-367, Mar. 1996.

[8] C. Scherer, P. Gahinet, and M. Chilali, "Multiobjective output-feedback control via LMI optimization," IEEE Trans. Automatic Control, Vol. 42, No. 7, pp. 896-911, 1997.

[9] P. Gahinet, A. Nemirovski, A. J. Laub, and M. Chilali, LMI control toolbox for use with MATLAB, The Mathworks Inc., 2000.

[10] R. Y. Chiang and M. G. Safonov, Robust control toolbox for use with MATLAB, The Mathworks Inc., 1996.

[11] E. Omine, T. Senjyu, "Torsional torque suppression of decentralized generators based on $H_{\infty}$ control theory," Proceedings of the 2006 Joint Conference of the IEEJ and IEICE, pp. 151-156, Dec. 2008.

[12] Zenta Iwai, Akira Inoue, Shigeyasu Kawazi, Observer, CORONA PUBLISHING CO., LTD., 1990.

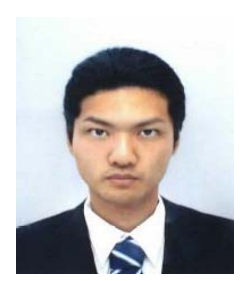

Tomonori Goya was born in Okinawa, Japan, in 1988. He received his B.S. from the Department of Electrical and Electronics Engineering, Faculty of Engineering, University of the Ryukyus. He is currently working toward his M.S. in the Department of Electrical and Electronics Engineering, Faculty of Engineering, University of the Ryukyus. His research interests include system operation of transmission systems. Mr. Goya is a student member of the Institute of Electrical and Electronics Engineers and the Institute of Electrical Engineers of Japan.

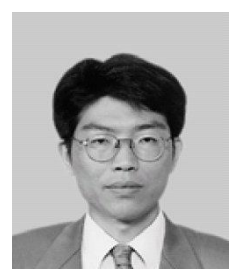

Tomonobu Senjyu was born in Saga Prefecture, Japan, in 1963. He received his B.S. and M.S. in Electrical Engineering from the University of the Ryukyus, Okinawa, Japan, in 1986 and 1988, respectively, and his Ph.D. in Electrical Engineering from Nagoya University, Nagoya, Japan, in 1994. Since 1988, he has been with the Department of Electrical and Electronics Engineering, Faculty of Engineering, University of the Ryukyus, where he is currently a Professor. His research interests are in the areas of stability of ac machines, advanced control of electrical machines, and power electronics. Prof. Senjyu is a member of the Institute of Electrical Engineers of Japan.

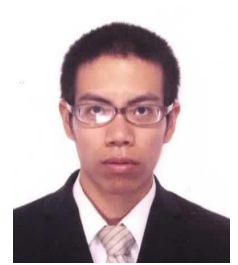

Eitaro Omine (Student Member) was born in Okinawa, Japan, in 1984. He received his B.S. from the University of the Ryukyus, Okinawa, Japan, in 2007, in Electrical and Electronics Engineering. He is currently pursuing his M.S. in the Department of Electrical and Electronics Engineering, Faculty of Engineering, University of the Ryukyus. His research interests include stable operation of dispersed generators, and stability and control of power systems. Mr. Omine is a student member of the Institute of Electrical and Electronics Engineers and the Institute of Electrical Engineers of Japan.

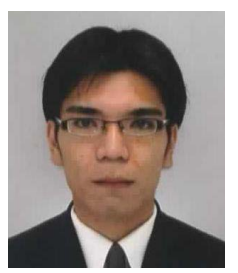

Atsushi Yona (S'06) was born in Okinawa, Japan, in 1982. He received his B.S. in Electrical Engineering from the University of the Ryukyus, Okinawa, Japan, in 2006. He is currently pursing his M.S. at the University of the Ryukyus. His research interests include wind and photovoltaic energies, and neural networks. Mr. Yona is a student member of the IEEE and the IEEJ.

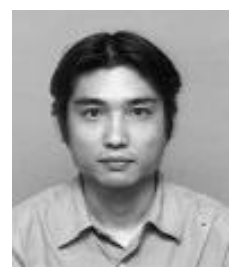

Naomitsu Urasaki was born in Okinawa Prefecture, Japan in 1973. He received his B.S. and M.S. in Electrical Engineering from the University of the Ryukyus, Japan, in 1996 and 1998, respectively. Since 1998, he has been with Department of Electrical and Electronics Engineering, Faculty of Engineering, University of the Ryukyus, where he is currently a Research Associate. His research interests are in the areas of modeling and control of ac motors. Mr. Urasaki is a Member of the Institute of Electrical Engineers of Japan.

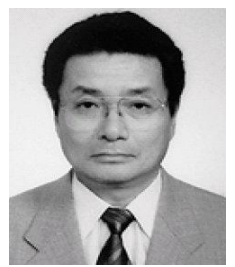

Toshihisa Funabashi (M'90-SM'96) was born in Aichi, Japan, in 1951. He received his B.S. in Electrical Engineering from Nagoya University, Aichi, Japan, in 1975. He received his Ph.D. in Electrical Engineering from Doshisha University, Kyoto, Japan, in 2000. In 1975, he joined the Meidensha Corporation, Tokyo, Japan, where he was engaged in research on power system analysis and is currently the Senior Engineer of the Power System Engineering Division. Dr. Funabashi is a chartered engineer in the U.K. and a member of the IEEE and the IEET of Japan. 